\title{
Tratamento do herpes zoster e prevenção da nevralgia pós-herpética
}

Joana Gonçalves Luís, ${ }^{1}$ Bárbara Martins ${ }^{1}$

\section{RESUMO}

Objetivos: A nevralgia pós-herpética (NPH) é a principal complicação do herpes zoster (HZ), tendo um impacto significativo na qualidade de vida dos doentes e nos custos em saúde. Com esta revisão pretende-se avaliar a eficácia de diferentes terapêuticas utilizadas no tratamento agudo da zona na prevenção desta complicação.

Fontes de dados: MEDLINE/PubMed, National Guide Clearinghouse, Canadian Medical Association Practice Guidelines, Bandolier, Evidence-Based Medicine Online, DARE, TRIP Database e The Cochrane Library.

Métodos de revisão: Pesquisa de guidelines, meta-análises, revisões sistemáticas e ensaios clínicos controlados e aleatorizados publicados entre abril de 2010 e março de 2020, redigidos em inglês, português ou espanhol, utilizando os termos MeSH herpes zoster e neuralgia, postherpetic.

Resultados: Dos 333 resultados obtidos na pesquisa inicial foram selecionados sete artigos para inclusão nesta revisão, nomeadamente duas meta-análises, uma revisão sistemática e quatro ensaios clínicos controlados e aleatorizados. A evidência encontrada relativamente à utilização de antivíricos, glucocorticoides ou gabapentinoides no tratamento do HZ não demonstrou benefício na prevenção da NPH. Apenas as opções por amitriptilina ou vitamina $\mathrm{C}$ endovenosa revelaram resultados positivos, ainda que com evidência escassa e limitada.

Conclusão: Esta revisão permite concluir que nenhum dos tratamentos habitualmente utilizados no tratamento agudo do $\mathrm{HZ}$ previne a ocorrência de NPH. Apesar dos resultados positivos da amitriptilina e da vitamina C não existe ainda evidência suficientemente robusta que permita recomendar o uso disseminado destes fármacos na fase aguda do $\mathrm{HZ}$ com o intuito de prevenir esta complicação. Mais estudos são necessários neste sentido, tendo em conta a morbilidade e custos em saúde associados à NPH.

Palavras-chave: Herpes zoster; Zona; Nevralgia pós-herpética.

\section{INTRODUÇÃO}

A zona é uma doença cutâneo-sensorial resultante da reativação do vírus varicela zoster (VZV), devido à sua latência nos gânglios nervosos sensitivos após a infeção primária causadora de varicela. ${ }^{1}$ A reativação ocorre em aproximadamente $25 \%$ dos indivíduos, ${ }^{2}$ causando uma erupção cutânea dolorosa característica, que geralmente afeta apenas um dermátomo, inicialmente com lesões maculares, progressão para vesículas e pústulas e posterior formação de crostas, seguida de cicatrização. ${ }^{3}$ Os dermátomos mais frequentemente atingidos são o torácico, trigeminal, lombar e cervical, maioritariamente de forma uni-

1. USF São Lourenço, ACeS do Cávado I. Braga, Portugal. lateral e sem cruzamento da linha média, ainda que qualquer área da pele possa ser afetada. ${ }^{1} \mathrm{O}$ quadro cutâneo pode ser precedido de sintomas prodrómicos, como prurido, disestesias e dor local intensa, desde 2 a 18 dias antes do surgimento da erupção cutânea. ${ }^{3}$

Estima-se que cerca de um em cada três indivíduos terá pelo menos um episódio de zona ao longo da vida, ${ }^{4}$ com uma incidência anual na Europa a variar entre $2 \mathrm{a}$ 4,6 casos de herpes zoster (HZ) por 1000 pessoas por ano, sem diferenças geográficas significativas. ${ }^{5}$ Verifica-se um incremento da incidência com a idade em estreita relação com o declínio da imunidade celular, particularmente a partir dos 50 anos, podendo atingir os $50 \%$ na faixa etária dos 85 anos. ${ }^{6}$ Para além da idade como fator de risco para o desenvolvimento de $\mathrm{HZ}$ 
destacam-se ainda a imunossupressão, na qual se inclui a infeção pelo vírus da imunodeficiência humana (VIH), transplantação, neoplasias e outras doenças com necessidade de tratamento imunossupressor, género feminino, raça caucasiana e trauma físico precedente. ${ }^{7}$

A nevralgia pós-herpética (NPH) constitui a principal complicação do HZ, definindo-se tradicionalmente como uma dor significativa de caráter neuropático no dermátomo afetado, que persiste após 90 dias do início das lesões cutâneas como resultado do dano nervoso induzido pelo vírus. ${ }^{8} \mathrm{~A}$ sua incidência é variável entre estudos, num intervalo de 5 a $30 \%,{ }^{9}$ sendo fatores de risco conhecidos para o desenvolvimento de NPH a idade avançada, dor severa na fase aguda, ocorrência de pródromo e a presença de lesões cutâneas graves. ${ }^{10-11}$ Portadores de doenças crónicas, como é o caso de diabéticos e imunodeprimidos, podem apresentar maior gravidade da doença. ${ }^{12} \mathrm{~A}$ dor pode persistir durante meses ou anos e afetar negativamente a qualidade de vida dos doentes, ${ }^{13}$ existindo vários estudos publicados relativamente ao impacto económico significativo desta condição em diferentes populações. ${ }^{14-16}$ Pode ser de tal forma incapacitante ao ponto de se associar a depressão, fadiga, insónia, anorexia, inatividade física, diminuição da capacidade de concentração e isolamento social. ${ }^{17}$

O tratamento da zona tem como objetivos principais diminuir a extensão e duração dos sintomas cutâneos, assim como a gravidade da dor aguda associada e prevenir as complicações da doença. ${ }^{3}$ Os antivíricos sistémicos constituem a base do tratamento, associando-se o uso de analgésicos para o controlo da nevrite aguda. ${ }^{1}$ Os antivíricos análogos dos nucleosídeos são a primeira escolha no tratamento da zona, estando recomendado o seu uso preferencialmente nas primeiras 72 horas de aparecimento das lesões cutâneas ou mais tardiamente na presença de situações específicas, entre as quais a imunossupressão, o surgimento de novas vesículas, HZ oftálmico ou HZ ótico e disseminação cutânea, visceral ou neurológica. ${ }^{3,18}$ Outros agentes podem ser ainda considerados como opções terapêuticas, dependendo da situação clínica, como os glucocorticoides. ${ }^{3}$ No campo da prevenção existem já duas vacinas efetivas contra o HZ, apenas uma delas disponível em Portugal, estando demonstrado o seu benefício e efetividade na redução da incidência da doença e da morbilidade associada. ${ }^{19} \mathrm{O}$ objetivo desta revisão foi procurar evidência acerca da efetividade dos fármacos utilizados no tratamento da zona na prevenção da NPH, tendo em conta a morbilidade associada a esta condição que afeta muito significativamente a qualidade de vida dos doentes.

\section{MÉTODOS}

Para a elaboração desta revisão procedeu-se a uma pesquisa bibliográfica referente a artigos publicados entre abril de 2010 e março de 2020, utilizando os termos MeSH herpes zoster e neuralgia, postherpetic, nas bases de dados: MEDLINE/PubMed, National Guide Clearinghouse, Canadian Medical Association Practice Guidelines, Bandolier, Evidence-Based Medicine online, DARE, TRIP Database e The Cochrane Library. Foram incluídos artigos em Português, Inglês ou Espanhol, realizados em humanos, do tipo meta-análises, revisões sistemáticas, ensaios clínicos controlados e aleatorizados (ECA) e guidelines. A inclusão dos artigos na revisão baseou-se nos seguintes critérios, de acordo com o modelo PICO: ${ }^{20}$ População - adultos imunocompetentes com HZ agudo; Intervenção - tratamento com agentes farmacológicos de administração oral ou endovenosa; Comparação - placebo ou não tratamento; Outcome-incidência de NPH. Foram excluídos artigos que não fossem de encontro ao objetivo da revisão ou que não cumprissem estes pressupostos. Estudos referentes a outras modalidades terapêuticas, nomeadamente formulações tópicas no caso do HZ oftálmico e bloqueios nervosos ou estimulação por radiofrequência, foram igualmente excluídos.

\section{RESULTADOS E DISCUSSÃO}

Na pesquisa inicial obtiveram-se 333 resultados, dos quais apenas sete cumpriam os critérios de inclusão. A revisão incluiu assim duas meta-análises, uma revisão sistemática e quatro ensaios clínicos controlados e aleatorizados. O processo de seleção dos artigos encontra-se pormenorizado na Figura 1. Os resultados encontram-se sumarizados nas Tabelas 1 a 5, organizados por classe farmacológica e de acordo com o tipo de estudo.

\section{Antivíricos}

Os antivíricos constituem o tratamento de primeira linha da infeção por HZ, limitando a gravidade da erupção cutânea e acelerando a resolução da nevrite aguda em comparação com o placebo. ${ }^{21}$ São ainda essenciais na 


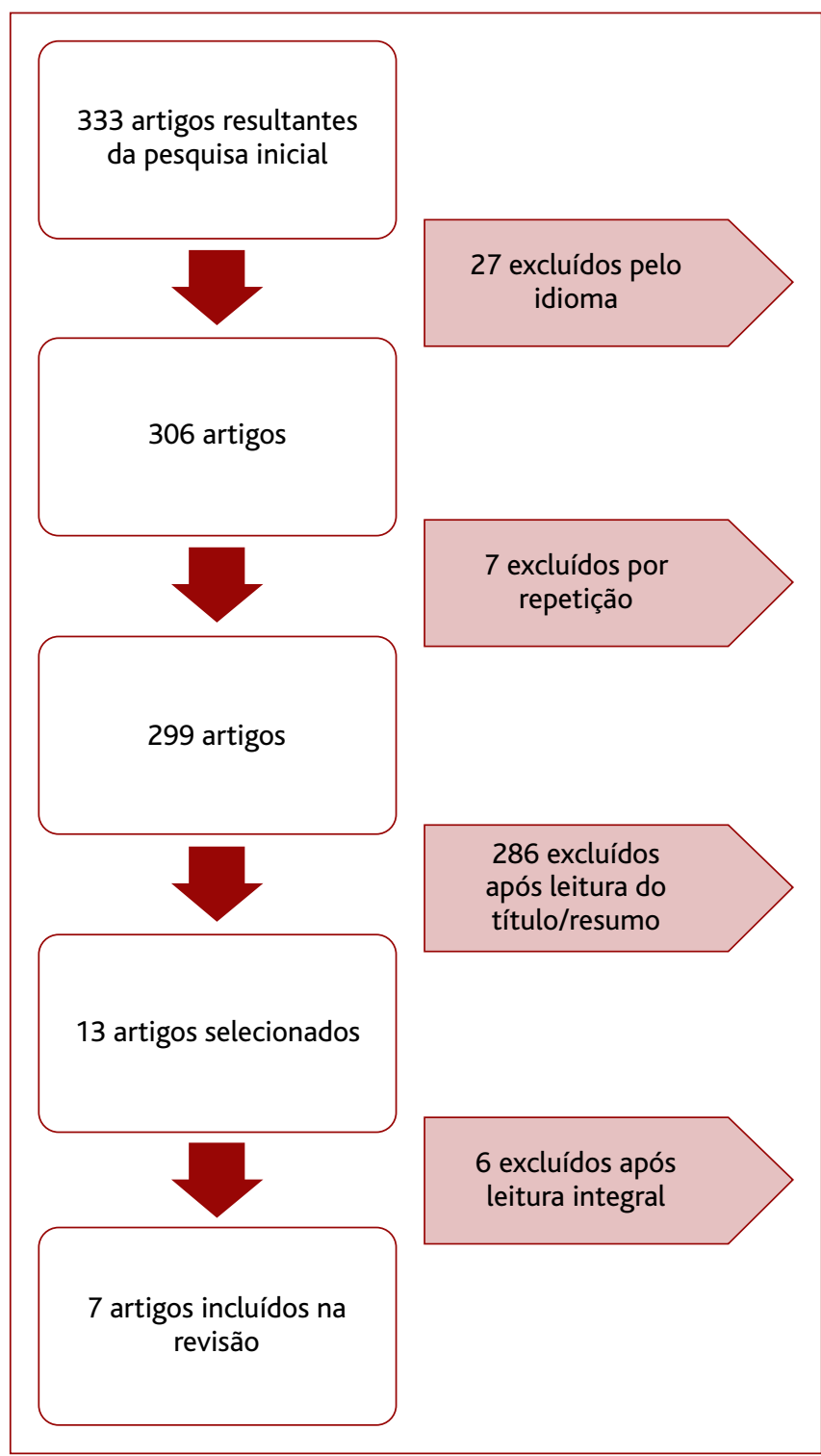

Figura 1. Fluxograma de seleção dos artigos.

prevenção de várias complicações da doença, como por exemplo a ocorrência de danos oculares decorrentes do $\mathrm{HZ}$ oftálmico. ${ }^{21} \mathrm{O}$ impacto da sua utilização na prevenção da NPH tem vindo a ser estudado por alguns autores, muitas vezes com resultados contraditórios entre os estudos. Na presente revisão foi contemplada uma meta-análise de Chen e colaboradores ${ }^{22}$ relativa ao uso desta classe de fármacos, sendo que nenhum dos estudos incluídos demonstrou redução estatisticamente significativa na ocorrência de NPH. A publicação incluiu cinco ar- tigos, todos datados anteriormente a 2000, comparando o uso de aciclovir ou famciclovir com placebo, em regimes terapêuticos de duração variável entre 7 a 21 dias. A própria definição de NPH diferiu entre os estudos, no que respeita ao intervalo de tempo decorrido entre o surgimento das lesões cutâneas e a manutenção da dor, variando entre quatro e seis meses. Dado o número reduzido de estudos englobados e das consideráveis diferenças entre os regimes terapêuticos e definição de NPH utilizada, a evidência existente permanece parca para suportar o papel dos antivíricos na redução da incidência desta complicação da doença, apesar dos seus benefícios na fase aguda. No que respeita a outros antivíricos frequentemente utilizados na prática clínica, nomeadamente o valaciclovir e a brivudina, não foram encontrados estudos incluindo a sua utilização.

\section{Glucocorticoides}

Os glucocorticoides são uma arma terapêutica muito versátil, sendo também considerados de forma controversa como adjuvantes no tratamento do HZ pelo seu potencial em reduzir a dor aguda, em acelerar a resolução das lesões e a melhorar o retorno às atividades habituais. ${ }^{1,11}$ Não devem ser utilizados isoladamente dos antivíricos, sendo que em formulações tópicas exibem um papel importante no HZ oftálmico. ${ }^{12} \mathrm{~A}$ sua eficácia na prevenção da NPH foi analisada por uma meta-análise de Han e colaboradores, ${ }^{23}$ que incluiu dois ensaios clínicos prévios a 1990. O uso de glucocorticoides durante 21 dias, com início nos primeiros sete dias das lesões cutâneas, em associação ou não com antivíricos, não se revelou eficaz na prevenção da NPH aos seis meses em comparação com o placebo.

\section{Gabapentinoides}

Os gabapentinoides fazem parte das opções terapêuticas para o tratamento da dor neuropática, pelo que a sua aplicação no tratamento da nevrite aguda associada à infeção por HZ pode ser considerada. ${ }^{1,18}$ Esta classe farmacológica está também recomendada no tratamento específico da NPH. ${ }^{8} \mathrm{Na}$ presente revisão foram incluídos três ensaios clínicos relativos ao uso destes fármacos, sendo que nenhum deles demonstrou impacto estatisticamente significativo na prevenção da 


\begin{tabular}{|c|c|c|c|c|c|c|}
\hline \multicolumn{7}{|c|}{ ANTIVÍRICOS } \\
\hline \multicolumn{7}{|c|}{ Meta-análises } \\
\hline $\begin{array}{l}\text { Autor } \\
\text { e Ano }\end{array}$ & $\begin{array}{l}\text { Fármaco } \\
\text { em estudo }\end{array}$ & $\begin{array}{l}N^{\circ} \text { ECA } \\
\text { incluídos }\end{array}$ & Amostra & $\begin{array}{l}\text { Outcome } \\
\text { estudado }\end{array}$ & Intervenção & Resultados \\
\hline \multirow[t]{3}{*}{$\begin{array}{l}\text { Chen, } \\
\text { et al. } \\
(2014)^{22}\end{array}$} & $\begin{array}{l}\text { Aciclovir } \\
\text { oral }\end{array}$ & 2 & $\begin{array}{l}n=476 \\
\text { (apresentação } \\
\text { nas primeiras } \\
72 \mathrm{~h} \text { da erupção } \\
\text { cutânea) }\end{array}$ & $\begin{array}{l}\text { Presença de NPH } \\
\text { após } 6 \text { meses do } \\
\text { início das lesões } \\
\text { cutâneas }\end{array}$ & $\begin{array}{l}\text { Wood (1988): } \\
\text { - Aciclovir 800mg 5x/dia } \\
\text { vs placebo durante } 7 \text { dias } \\
\text { Whitley (1996): } \\
\text { - Aciclovir 800mg 5x/dia + } \\
\text { prednisona vs aciclovir } \\
\text { 800mg 5x/dia + placebo vs } \\
\text { prednisona + placebo vs } \\
\text { placebo + placebo durante } \\
21 \text { dias }\end{array}$ & $\begin{array}{l}\text { O tratamento com } \\
\text { aciclovir não preveniu } \\
\text { significativamente a } \\
\text { ocorrência de NPH } \\
\text { (RR=1,05; IC95\% } \\
0,87-1,27 ; p=0,72 \text { ) }\end{array}$ \\
\hline & $\begin{array}{l}\text { Aciclovir } \\
\text { oral }\end{array}$ & 3 & $\begin{array}{l}n=609 \\
\text { (apresentação } \\
\text { nas primeiras } \\
72 \mathrm{~h} \text { da erupção } \\
\text { cutânea) }\end{array}$ & $\begin{array}{l}\text { Presença de NPH } \\
\text { após } 4 \text { meses do } \\
\text { início das lesões } \\
\text { cutâneas }\end{array}$ & $\begin{array}{l}\text { Wood (1988): } \\
\text { - Aciclovir 800mg 5x/dia } \\
\text { vs placebo durante } 7 \text { dias } \\
\text { Huff (1988): } \\
\text { - Aciclovir } 800 \mathrm{mg} 5 x / \text { dia vs } \\
\text { placebo durante } 10 \text { dias } \\
\text { Harding (1991): } \\
\text {-Aciclovir } 800 \mathrm{mg} 5 x / \text { dia vs } \\
\text { placebo durante } 10 \text { dias }\end{array}$ & $\begin{array}{l}\text { A incidência de NPH foi } \\
\text { menor no grupo tratado } \\
\text { com aciclovir vs. placebo, } \\
\text { porém de forma não } \\
\text { estatisticamente } \\
\text { significativa } \\
\text { (RR=0,75; IC95\% } \\
0,51-1,11 ; p=0,15)\end{array}$ \\
\hline & $\begin{array}{l}\text { Famciclovir } \\
\text { oral }\end{array}$ & 1 & $\begin{array}{l}n=419 \\
\text { (apresentação } \\
\text { nas primeiras } \\
72 \mathrm{~h} \text { da erupção } \\
\text { cutânea) }\end{array}$ & $\begin{array}{l}\text { Presença de NPH } \\
\text { após } 5 \text { meses } \\
\text { desde a resolução } \\
\text { das lesões } \\
\text { cutâneas }\end{array}$ & $\begin{array}{l}\text { Tyring (1995): } \\
\text { - Famciclovir 500mg ou } \\
750 \mathrm{mg} \text { vs placebo durante } \\
7 \text { dias }\end{array}$ & $\begin{array}{l}\text { O tratamento com } \\
\text { famciclovir não preveniu } \\
\text { de forma estatisticamente } \\
\text { significativa a ocorrência } \\
\text { de } \mathrm{NPH} \\
\text { (Dose } 500 \mathrm{mg}: \mathrm{RR}=1,15 ; \\
\text { IC95\% }[0,87,1,52] \text {; dose } \\
750 \mathrm{mg}: \mathrm{RR}=1,31 \text {; IC95\% } \\
{[1,01,1,71] \text { ) }}\end{array}$ \\
\hline
\end{tabular}

NPH. Tanto no estudo multicêntrico de Buililete e

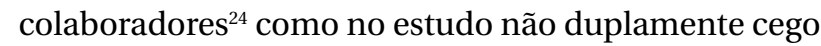
de Lee e colaboradores, ${ }^{25}$ o recurso à gabapentina durante a fase aguda e nas semanas seguintes não preveniu de forma estatisticamente significativa a ocorrência de NPH em comparação com o placebo ou com a não intervenção. No que concerne à opção por pregabalina foi incluído nesta revisão um estudo de pequena di- mensão de Škvar e colaboradores,$^{26}$ que comparou a utilização deste fármaco com o placebo na prevenção de NPH aos seis meses e que também não demonstrou benefício. Apesar da ausência de resultados positivos, nos três estudos citados os participantes do estudo receberam, para além da gabapentina, outros analgésicos não explícitos nos artigos, que podem de alguma forma introduzir um viés na atribuição do efeito antiálgico. 


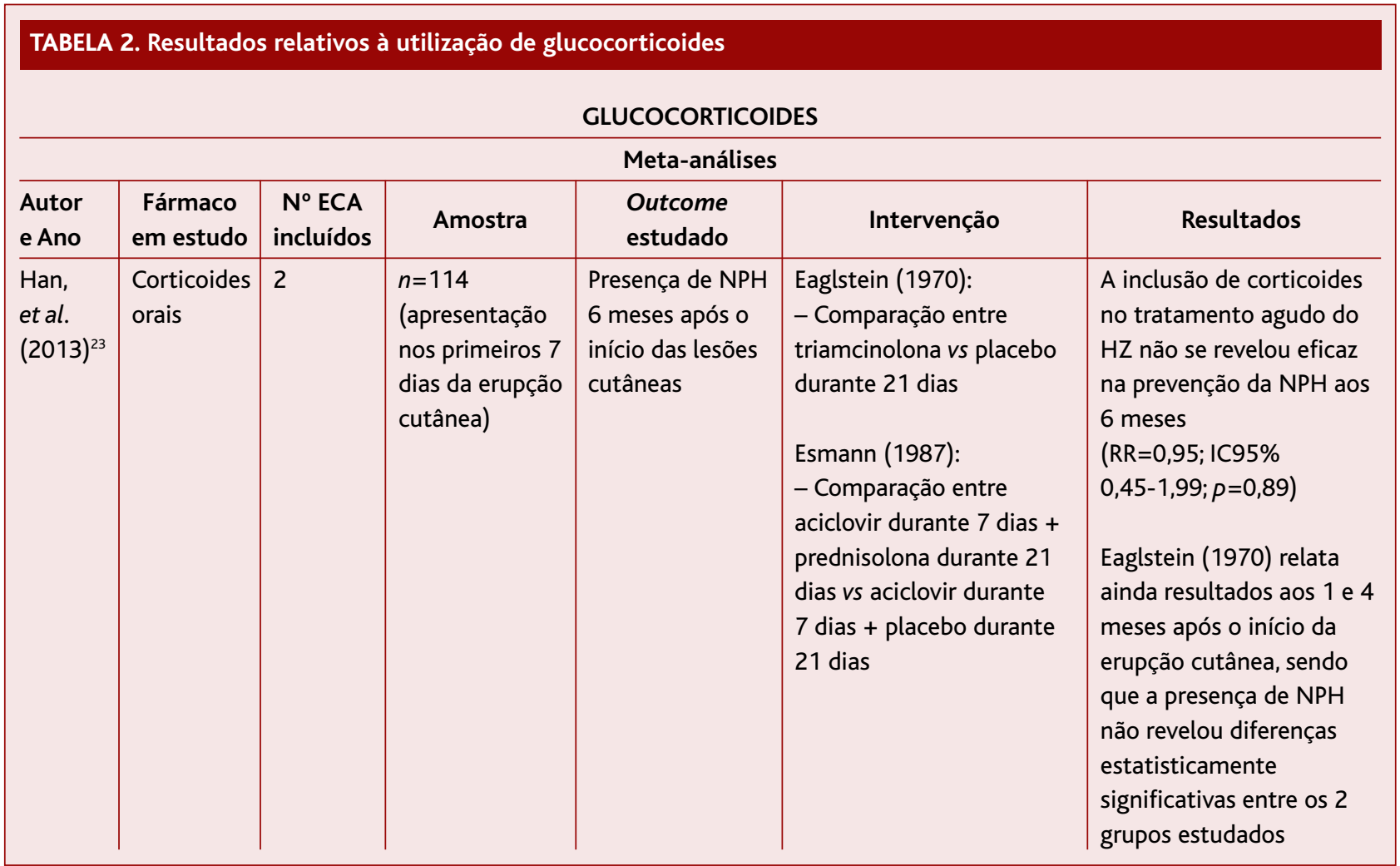

\section{Antidepressivos tricíclicos}

Tendo por base a utilização dos antidepressivos tricíclicos no tratamento da dor neuropática, a eficácia da inclusão desta classe farmacológica no tratamento da zona e consequente prevenção da NPH apresenta evidência escassa e controversa. Da pesquisa efetuada para efeitos desta revisão apenas foi encontrada uma revisão sistemática de Watson ${ }^{27} \mathrm{com}$ referência ao uso da amitriptilina na fase aguda do HZ. O único estudo incluído nessa revisão de 2010 reporta diferenças estatisticamente significativas entre o grupo tratado com amitriptilina $25 \mathrm{mg}$ por dia durante 90 dias $v s$. placebo no que respeita à ocorrência de NPH aos seis meses. Apesar do resultado positivo estão descritas várias limitações deste estudo, nomeadamente uma significância estatística borderline, blinding questionável e ausência de racional para a prescrição ou não de antivíricos, tendo sido opção individual do médico a sua inclusão ou exclusão do regime terapêutico. A evidência relativa à eficácia dos antidepressivos tricíclicos na prevenção da NPH é, portanto, de baixa qualidade, sendo necessários mais estudos para confirmar este achado. De ressalvar ainda que esta classe farmacológica se associa a um leque de efeitos adversos que pode limitar o seu uso, sobretudo nos mais idosos. ${ }^{12}$

\section{Vitamina C}

Existe evidência de que a vitamina $C$ pode desempenhar um papel importante na prevenção e combate a determinadas infeções, desde a constipação comum até à pneumonia. ${ }^{28}$ Inclusivamente vários estudos reportam o seu potencial papel analgésico adjuvante no tratamento da dor em várias condições clínicas, nomeadamente em doentes oncológicos ou cirúrgicos. ${ }^{29}$ Para efeitos desta revisão foi incluído um ensaio clínico de 2016 levado a cabo por Kim e colaboradores, ${ }^{30}$ que utilizou o ácido ascórbico endovenoso como parte integrante do tratamento de doentes hospitalizados com HZ, juntamente com antivíricos e analgesia que incluiu a gabapentina. A administração de ácido ascórbico no primeiro, terceiro e quinto dias de tratamento associou-se a menor incidência de NPH às quatro 
TABELA 3. Resultados relativos à utilização de gabapentinoides

\section{GABAPENTINOIDES}

\begin{tabular}{|c|c|c|c|c|c|}
\hline \multicolumn{6}{|c|}{ Ensaios clínicos controlados e aleatorizados } \\
\hline $\begin{array}{l}\text { Autor } \\
\text { e Ano }\end{array}$ & $\begin{array}{c}\text { Fármaco } \\
\text { em estudo }\end{array}$ & Amostra & Outcome estudado & Intervenção & Resultados \\
\hline $\begin{array}{l}\text { Buililete, } \\
\text { et al. } \\
(2019)^{24}\end{array}$ & $\begin{array}{l}\text { Gabapentina } \\
\text { oral }\end{array}$ & $\begin{array}{l}n=98 \\
\text { (apresentação } \\
\text { com dor de } \\
\text { intensidade } \geq 4 \\
\text { nas primeiras } \\
72 \mathrm{~h} \text { da erupção } \\
\text { cutânea) }\end{array}$ & $\begin{array}{l}\text { Qualquer grau de } \\
\text { dor }>0 \text { presente na } \\
12^{\mathrm{a}} \text { semana após a } \\
\text { erupção cutânea }\end{array}$ & $\begin{array}{l}\text { Estudo multicêntrico } \\
\text { Todos os participantes foram } \\
\text { tratados com valaciclovir } \\
\text { 1000mg 3x/dia durante } 7 \text { dias } \\
\text { e com um analgésico não } \\
\text { especificado, de acordo com a } \\
\text { escada de tratamento da dor } \\
\text { da OMS. } \\
\text { - Grupo controlo: placebo } \\
\text { - Grupo de estudo: } \\
\text { gabapentina } 300 \text { mg/dia (com } \\
\text { um incremento diário da dose } \\
\text { de } 300 \mathrm{mg} \text { até ao teto máximo } \\
\text { de } 1800 \mathrm{mg} / \text { dia no } 7^{\circ} \text { dia de } \\
\text { tratamento; diminuição da } \\
\text { dose na última semana) } \\
\text { Duração do tratamento: } 5 \\
\text { semanas }\end{array}$ & 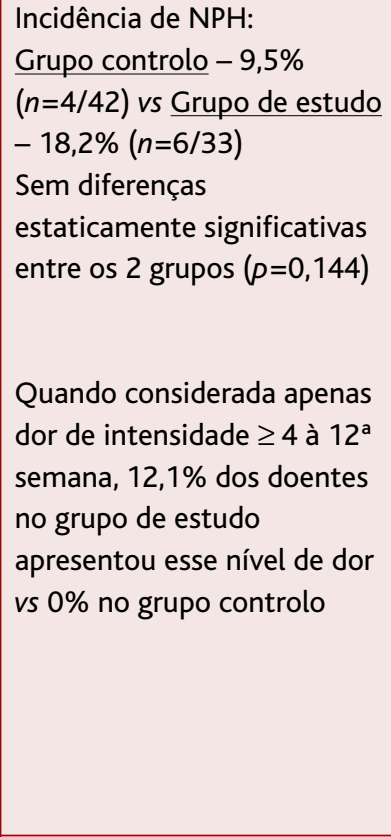 \\
\hline $\begin{array}{l}\text { Lee, et al. } \\
(2016)^{25}\end{array}$ & $\begin{array}{l}\text { Gabapentina } \\
\text { oral }\end{array}$ & $\begin{array}{l}n=120 \\
\text { (apresentação } \\
\text { com dor de } \\
\text { intensidade } \geq 4 \\
\text { nos primeiros } 4 \\
\text { dias da erupção } \\
\text { cutânea) }\end{array}$ & $\begin{array}{l}\text { Existência de dor } \\
\text { moderada a severa } \\
\text { (intensidade } \geq 4 \text { ) } \\
\text { após } 12 \text { semanas do } \\
\text { tratamento inicial }\end{array}$ & $\begin{array}{l}\text { Estudo não duplamente cego } \\
\text { Todos os participantes foram } \\
\text { tratados com valaciclovir } \\
\text { 3000mg/dia durante } 1 \\
\text { semana e acetominofeno } \\
650 \mathrm{mg} 3 x / \text { dia } \\
\text { - Grupo controlo: sem } \\
\text { analgesia adicional } \\
\text { - Grupo de estudo: } \\
\text { gabapentina } 300 \mathrm{mg} 3 \times / \text { dia } \\
\text { durante o período de } \\
\text { persistência da dor até ao } \\
\text { máximo de } 12 \text { semanas (a } \\
\text { dose foi aumentada a cada } 3 \\
\text { dias até } 900 \mathrm{mg} / \text { dia) }\end{array}$ & 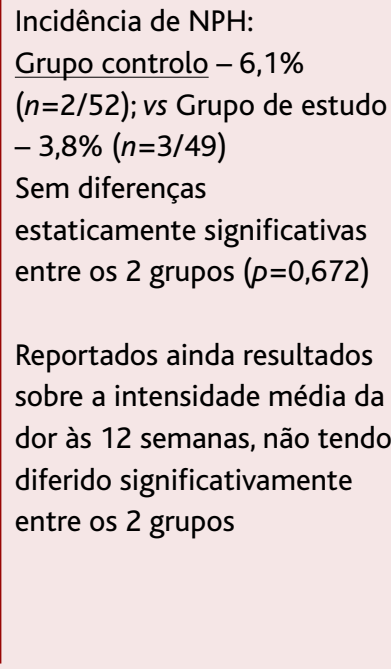 \\
\hline
\end{tabular}

semanas em comparação com o placebo. Apesar de este estudo ter definido NPH recorrendo ao período temporal mais curto de todos os trabalhos incluídos nesta revisão (quatro semanas), o que poderia fazer questionar os potenciais resultados da terapêutica numa fase mais tardia, são também reportados resultados positivos na $8^{\mathrm{a}}$ e $16^{\mathrm{a}}$ semanas, com diferenças estatisticamente significativas comparativamente ao grupo controlo. 


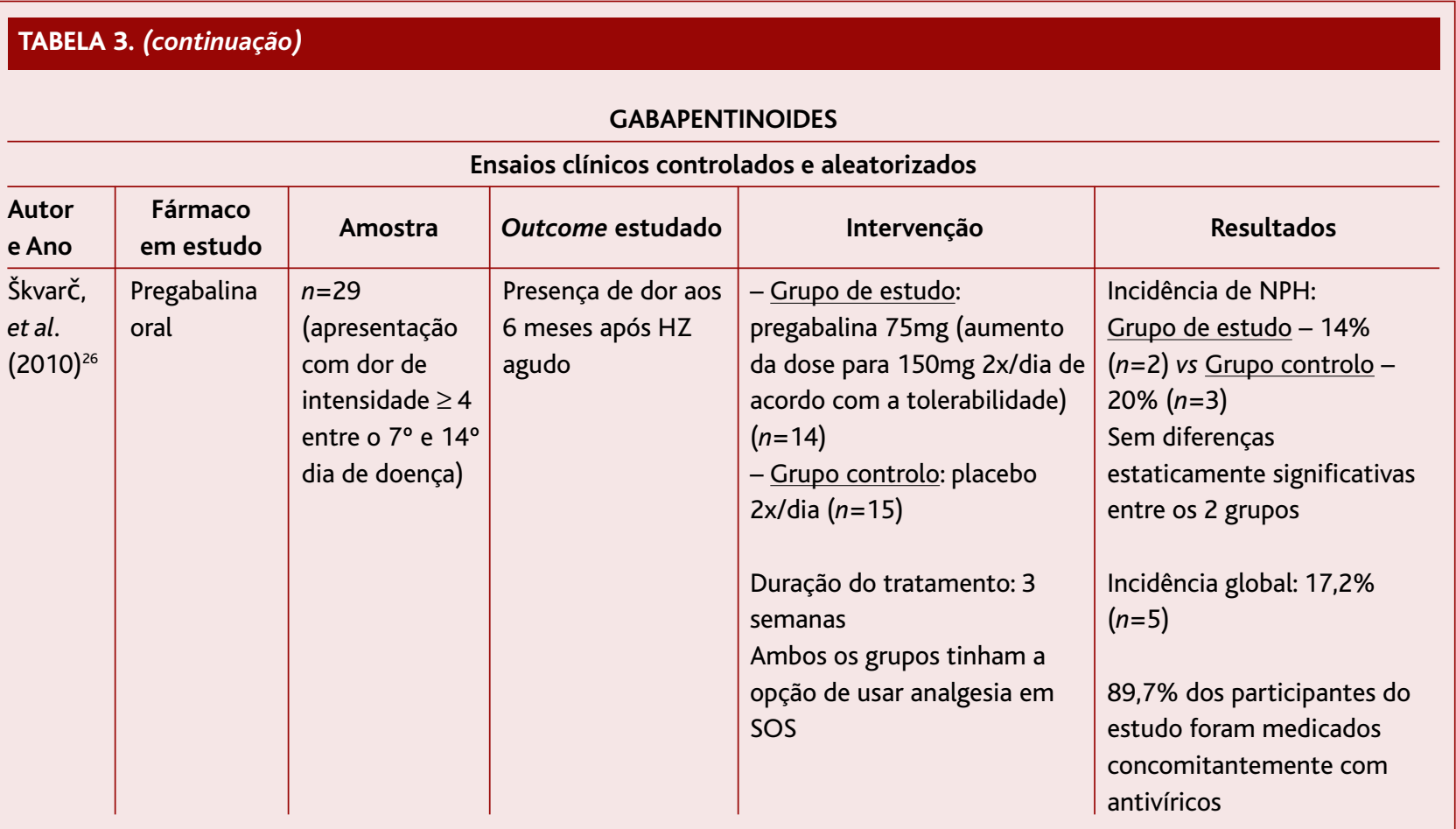

TABELA 4. Resultados relativos à utilização de antidepressivos tricíclicos

\begin{tabular}{|c|c|c|c|c|c|c|}
\hline \multicolumn{7}{|c|}{ ANTIDEPRESSIVOS TRICÍCLICOS } \\
\hline \multicolumn{7}{|c|}{ Revisões sistemáticas } \\
\hline $\begin{array}{l}\text { Autor } \\
\text { e Ano }\end{array}$ & $\begin{array}{c}\text { Fármaco } \\
\text { em estudo }\end{array}$ & $\begin{array}{l}N^{\circ} \text { ECA } \\
\text { incluídos }\end{array}$ & Amostra & $\begin{array}{l}\text { Outcome } \\
\text { estudado }\end{array}$ & Intervenção & Resultados \\
\hline $\begin{array}{l}\text { Watson } \\
(2010)^{27}\end{array}$ & $\begin{array}{l}\text { Amitriptilina } \\
\text { oral }\end{array}$ & 1 & $\begin{array}{l}n=80 \\
\text { (apresentação } \\
\text { nas primeiras } \\
48 \mathrm{~h} \text { da erupção } \\
\text { cutânea) }\end{array}$ & $\begin{array}{l}\text { Presença de } \\
\text { NPH } 6 \text { meses } \\
\text { após o início } \\
\text { das lesões } \\
\text { cutâneas }\end{array}$ & $\begin{array}{l}\text { - Grupo de estudo: } \\
\text { amitriptilina } 25 \mathrm{mg} 1 \times \text { /dia } \\
\text { - Grupo controlo: placebo } \\
\text { Duração do tratamento: } 90 \\
\text { dias } \\
\text { Prescrição de antivíricos de } \\
\text { acordo com a opção do } \\
\text { médico }\end{array}$ & $\begin{array}{l}\text { Ausência de dor aos } 6 \\
\text { meses: } \\
\text { Grupo de estudo - } 84 \% \\
(n=32 / 28) \text { vs Grupo } \\
\text { controlo }-65 \%(n=22 / 34) \\
\text { Com diferenças } \\
\text { estatisticamente } \\
\text { significativas entre os } 2 \\
\text { grupos ( } p<0,05) \text {, porém de } \\
\text { significância borderline de } \\
\text { acordo com o autor da RS }\end{array}$ \\
\hline
\end{tabular}

Embora este estudo não tenha ocorrido de forma duplamente cega, pois os investigadores tinham conhecimento se estavam a administrar placebo ou ácido ascórbico, os resultados parecem promissores e reforçam a necessidade de desenvolver mais estudos neste sentido. A vitamina C é economicamente acessível e facilmente disponível, pelo que o seu potencial impacto positivo na prevenção desta complicação é de considerar. 
TABELA 5. Resultados relativos à utilização de vitamina C

VITAMINA C

Ensaios clínicos controlados e aleatorizados

\begin{tabular}{|c|c|c|c|c|c|}
\hline $\begin{array}{l}\text { Autor } \\
\text { e Ano }\end{array}$ & $\begin{array}{c}\text { Fármaco } \\
\text { em estudo }\end{array}$ & Amostra & Outcome estudado & Intervenção & Resultados \\
\hline $\begin{array}{l}\text { Kim, } \\
\text { et al. } \\
(2016)^{30}\end{array}$ & $\begin{array}{l}\text { Ácido } \\
\text { ascórbico } \\
\text { endovenoso } \\
\text { (EV) }\end{array}$ & $\begin{array}{l}n=87 \\
\text { (doentes } \\
\text { hospitalizados } \\
\text { por HZ no } \\
\text { serviço de } \\
\text { dermatologia) }\end{array}$ & $\begin{array}{l}\text { NPH definida como } \\
\text { score de dor às } 4 \\
\text { semanas, após o } \\
\text { início das lesões } \\
\text { cutâneas superior a } \\
30 \% \text { do score de } \\
\text { dor inicial }\end{array}$ & 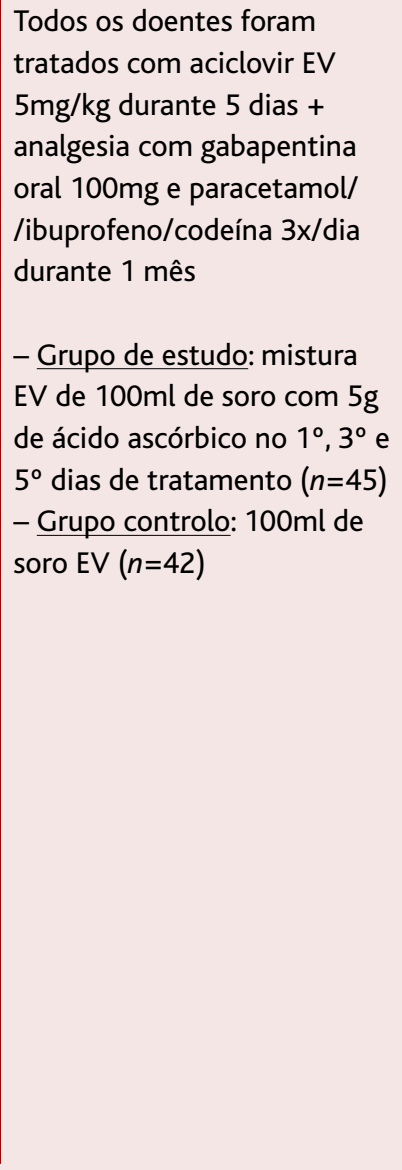 & 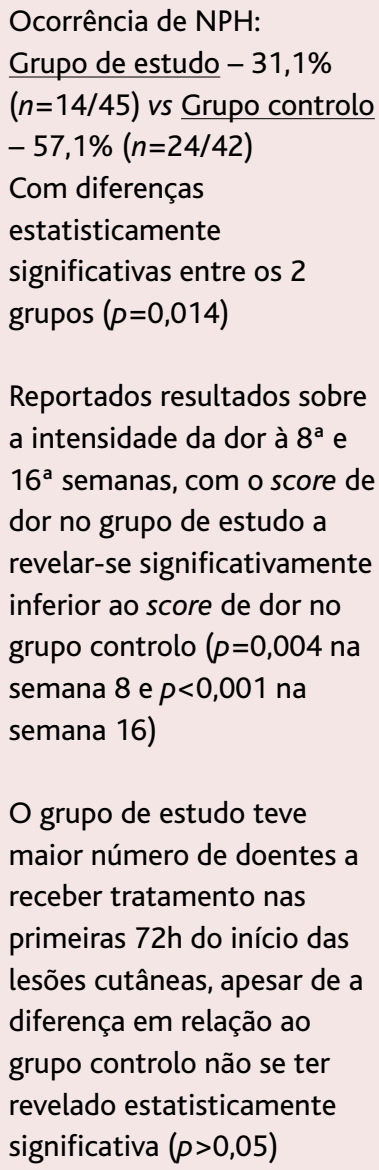 \\
\hline
\end{tabular}

\section{CONCLUSÃO}

A NPH constitui a complicação mais comum do HZ, estando associada a um impacto negativo significativo na qualidade de vida dos doentes e a elevados custos em saúde. Apesar das várias classes terapêuticas disponíveis e efetivas no tratamento agudo da zona e da nevrite associada, a evidência existente revela-se insuficiente para sustentar o benefício do seu uso na prevenção da NPH. Os estudos referentes à amitriptilina e à vitamina $\mathrm{C}$ foram os únicos com resultados positivos; porém, apresentam limitações evidentes dada a baixa potência estatística e ausência de reprodutibilidade dos resultados. O número de trabalhos incluídos na presente revisão é reduzido, com grande variabilidade nas metodologias utilizadas, tornando necessários mais estudos neste sentido, inclusivamente com recurso a fármacos de utilização comum na prática clínica atual, tendo em conta a morbilidade e custos em saúde subjacentes a esta patologia. A dor da NPH pode ser incapacitante e de muito difícil tratamento, pelo que justificaria um maior investimento na procura de um tratamento eficaz que permita prevenir esta complicação 
debilitante. A sua prevenção parece passar, por enquanto, pela própria prevenção da reativação do vírus com recurso à utilização da vacina contra o $\mathrm{HZ}$, com particular interesse nos doentes em maior risco de desenvolver a doença e suas complicações. Ainda assim, o seu uso permanece limitado na prática clínica, devido ao custo económico associado e à escassa divulgação das suas indicações e benefícios entre a comunidade médica.

\section{REFERÊNCIAS BIBLIOGRÁFICAS}

1. Cohen JI. Herpes zoster. N Engl J Med. 2013;369(18):1766-7.

2. Wareham DW, Breuer J. Herpes zoster. BMJ. 2007;334(7605):1211-5.

3. Werner RN, Nikkels AF, Marinovic B, Schäfer M, Czarnecka-Operacz M, Agius AM, et al. European consensus-based (S2k) Guideline on the Management of Herpes Zoster: guided by the European Dermatology Forum (EDF) in cooperation with the European Academy of Dermatology and Venereology (EADV), Part 1: diagnosis. J Eur Acad Dermatol Venereol. 2017;31(1):9-19.

4. Gershon AA, Gershon MD, Breuer J, Levin MJ, Oaklander AL, Griffiths PD. Advances in the understanding of the pathogenesis and epidemiology of herpes zoster. J Clin Virol. 2010;48 Suppl 1:S2-7.

5. Pinchinat $\mathrm{S}$, Cebrián-Cuenca AM, Bricout $\mathrm{H}$, Johnson RW. Similar herpes zoster incidence across Europe: results from a systematic literature review. BMC Infect Dis. 2013;13:170.

6. Schmader K. Herpes zoster in older adults. Clin Infect Dis. 2001;32(10): 1481-6.

7. Marra F, Parhar K, Huang B, Vadlamudi N. Risk factors for herpes zoster infection: a meta-analysis. Open Forum Infect Dis. 2020;7(1): ofaa005.

8. Johnson RW, Rice AS. Postherpetic neuralgia. N Engl J Med. 2014;371 (16):1526-33.

9. Kawai K, Gebremeskel BG, Acosta CJ. Systematic review of incidence and complications of herpes zoster: towards a global perspective. BMJ Open. 2014;4(6):e004833.

10. Wei S, Li X, Wang H, Liu Q, Shao L. Analysis of the risk factors for postherpetic neuralgia. Dermatology. 2019;235(5):426-33.

11. Gan EY, Tian EA, Tey HL. Management of herpes zoster and post-herpetic neuralgia. Am J Clin Dermatol. 2013;14(2):77-85.

12. García-González Al, Rosas-Carrasco O. Herpes zoster and post-herpetic neuralgia in the elderly: particularities in prevention, diagnosis, and treatment. Gac Med Mex. 2017;153(1):92-101.

13. van Wijck AJ, Aerssens YR. Pain, itch, quality of life, and costs after herpes zoster. Pain Pract. 2017;17(6):738-46.

14. Schmidt-Ott R, Schutter U, Simon J, Nautrup BP, von Krempelhuber A, Gopala K, et al. Incidence and costs of herpes zoster and postherpetic neuralgia in German adults aged $\geq 50$ years: a prospective study. J Infect. 2018;76(5):475-82.

15. Matthews S, De Maria A, Passamonti M, Ristori G, Loiacono I, Puggina $A$, et al. The economic burden and impact on quality of life of herpes zoster and postherpetic neuralgia in individuals aged 50 years or older in Italy. Open Forum Infect Dis. 2019;6(2):ofz007.
16. Nilsson J, Cassel T, Lindquist L. Burden of herpes zoster and post-herpetic neuralgia in Sweden. BMC Infect Dis. 2015;15:215.

17. Schmader KE. Epidemiology and impact on quality of life of postherpetic neuralgia and painful diabetic neuropathy. Clin J Pain. 2002;18(6): 350-4.

18. Saguil A, Kane S, Mercado M, Lauters R. Herpes zoster and postherpetic neuralgia: prevention and management. Am Fam Physician. 2017; 96(10):656-63.

19. Chen N, Li Q, Zhang Y, Zhou M, Zhou D, He L. Vaccination for preventing postherpetic neuralgia. Cochrane Database Syst Rev. 2011;(3): CD007795.

20. Donato $H$, Donato M. Etapas na condução de uma revisão sistemática [Stages for undertaking a systematic review]. Acta Med Port. 2019; 32(3):227-35. Portuguese

21. Schmader K. Herpes zoster. Clin Geriatr Med. 2016;32(3):539-53.

22. Chen N, Li Q, Yang J, Zhou M, Zhou D, He L. Antiviral treatment for preventing postherpetic neuralgia. Cochrane Database Syst Rev. 2014;(2):CD006866.

23. Han Y, Zhang J, Chen N, He L, Zhou M, Zhu C. Corticosteroids for preventing postherpetic neuralgia. Cochrane Database Syst Rev. 2013;(3):CD005582.

24. Bulilete O, Leiva A, Rullán M, Roca A, Llobera J. Efficacy of gabapentin for the prevention of postherpetic neuralgia in patients with acute herpes zoster: a double blind, randomized controlled trial. PLoS One. 2019;14(6):e0217335.

25. Lee EG, Lee HJ, Hyun DJ, Min K, Kim DH, Yoon MS. Efficacy of low dose gabapentin in acute herpes zoster for preventing postherpetic neuralgia: a prospective controlled study. Dermatol Ther. 2016;29(3):184-90.

26. Skvarc NK, Kamenik M. Effects of pregabalin on acute herpetic pain and postherpetic neuralgia incidence. Wien Klin Wochenschr. 2010;122 Suppl 2:49-53.

27. Watson PN. Postherpetic neuralgia. BMJ Clin Evid. 2010;2010:0905.

28. Hemilä H. Vitamin C and infections. Nutrients. 2017;9(4):339.

29. Carr AC, McCall C. The role of vitamin $C$ in the treatment of pain: new insights. J Transl Med. 2017;15(1):77.

30. Kim MS, Kim DJ, Na CH, Shin BS. A study of intravenous administration of vitamin $C$ in the treatment of acute herpetic pain and postherpetic neuralgia. Ann Dermatol. 2016;28(6):677-83.

\section{CONFLITO DE INTERESSES}

As autoras declaram não ter quaisquer conflitos de interesse.

\section{FINANCIAMENTO}

As autoras declaram não ter recebido subsídios ou bolsas para a elaboração do artigo.

\section{ENDEREÇO PARA CORRESPONDÊNCIA}

Joana Gonçalves Luís

E-mail: joanagluis8@gmail.com

https://orcid.org/0000-0003-1487-4131

Recebido em 15-08-2020

Aceite para publicação em 01-09-2021 


\section{ABSTRACT}

\section{HERPES ZOSTER TREATMENT AND POSTHERPETIC NEURALGIA PREVENTION}

Objectives: Postherpetic neuralgia (PHN) is the main complication of herpes zoster ( $\mathrm{HZ})$, with a significant impact on patients' quality of life and health costs. The purpose of this review was to evaluate the efficacy of different therapies used in the acute treatment of shingles in the prevention of this complication.

Data sources: MEDLINE/PubMed, National Guide Clearinghouse, Canadian Medical Association Practice Guidelines, Bandolier, Evidence-Based Medicine Online, DARE, TRIP database, and The Cochrane Library.

Methods: Research of guidelines, meta-analyses, systematic reviews, and randomized and controlled clinical trials published between April 2010 and March 2020, written in English, Portuguese or Spanish, using the MeSH terms 'herpes zoster' and 'neuralgia, postherpetic'.

Results: From the 333 results obtained in the initial research, seven articles were selected to be included in this review, namely two meta-analyses, one systematic review, and four randomized and controlled clinical trials. Data found regarding the use of antivirals, glucocorticoids, or gabapentinoids in the treatment of $\mathrm{HZ}$ showed no benefit in the prevention of PHN. Only the option of amitriptyline or intravenous vitamin $\mathrm{C}$ revealed positive results, although with limited evidence.

Conclusion: This review concludes that none of the treatments commonly used in the acute treatment of HZ prevents PHN occurrence. Despite the positive results of amitriptyline and vitamin $C$, there is still insufficient evidence to recommend the disseminated use of these drugs in the acute phase of $\mathrm{HZ}$ in order to prevent this complication. Further studies are needed in this context considering the morbidity and health costs associated with PHN.

Keywords: Herpes zoster; Shingles; Postherpetic neuralgia. 